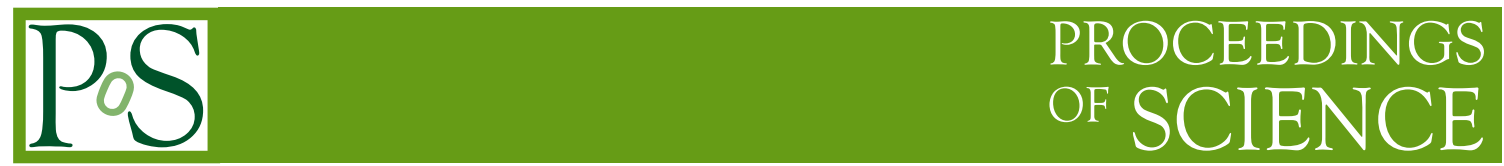

\title{
Jet environments
}

\section{James Miller-Jones* ${ }^{* \dagger}$}

National Radio Astronomy Observatory

E-mail: jmillerenrao.edu

While jets are launched and accelerated close to the compact object in X-ray binary systems, from that point onwards, their propagation and evolution is affected by the surrounding environment. Depending on the properties of the environment, they may inflate lobes of synchrotron-emitting plasma, sweep up shells of shock-compressed gas from the ambient medium, or create hotspots at the working surface where the jet impacts on the interstellar medium. Numerous examples of such phenomena have been detected in recent years, and I provide a review of the wide range of theoretical and observational work that has enhanced our understanding of the interaction of $\mathrm{X}$-ray binary jets with their environments. I finish by outlining the prospects for detecting more such interactions, and the effect that the upcoming generation of instruments (particularly the new radio facilities) will have on the field.

VII Microquasar Workshop: Microquasars and Beyond

September 1 - 5, 2008

Foca, Izmir, Turkey

*Speaker.

$\dagger$ Jansky Fellow, NRAO. 


\section{Introduction}

The interaction of the jets in active galactic nuclei (AGN) with the surrounding intergalactic medium (IGM) has been well studied for many years. Recent work on scaling relations between stellar mass and supermassive black holes, such as the so-called "Fundamental Plane of black hole activity" $[1,2]$, suggests that we might naively expect to see similar interactions of X-ray binary (XRB) jets with the surrounding interstellar medium (ISM). We have begun to find evidence for such interactions, and in this article, I will give an overview of the theoretical expectations for jetISM interactions, discuss some of the systems where interactions have been observed, summarize what we have learned from them, and highlight areas for further study with upcoming facilities.

When we consider the interaction of X-ray binary jets with their environments, we need to consider both the circumstellar environment and the local ISM through which the system is moving. High-mass X-ray binaries (HMXBs) are young systems, with powerful stellar winds which dominate their immediate surroundings. Being young, they can remain close to or within the boundaries of the supernova in which they were formed (SS 433, located within the W 50 nebula, is a prime example). Both the winds and the supernova ejecta will have a significant effect on the material through which the jets must propagate. Low-mass X-ray binaries (LMXBs), on the other hand, are older systems with less powerful winds. If they received any natal kick during their formation, they retain no association with their birthplace, and move relative to their local standard of rest (LSR), such that their immediate environment changes with time.

When comparing the environments of AGN jets with those of XRBs, we must recognise the intrinsic differences between the two types of system [3]. AGN are typically located in the centres of galaxy clusters. The cluster potential implies that the jets propagate through a stationary medium in which the density is decreasing monotonically away from the centre. The jets propagate outwards from the core of the system as highly-collimated beams, terminating at hotspots, which are the working surfaces where the jets impact on the IGM. Electrons are accelerated at these hotspots and flow back towards the core, creating diffuse lobes of old plasma which emit synchrotron radiation. XRBs, on the other hand, are moving through the Galactic potential, at velocities which can reach several hundred $\mathrm{km} \mathrm{s}^{-1}$ with respect to the LSR. Their environments are neither stationary nor centrally concentrated. Furthermore, comparing typical jet thrusts with the inertial density of the surroundings, we find that most XRB jets are located in relatively underdense, underpressured environments when compared to AGN jets [4].

\section{Theoretical expectations}

To explain such a counterintuitive statement, we have to consider the expected scaling of the quantities governing the large-scale dynamics of the jet, following the arguments set out by [4]. For active jets, the dynamics are governed by the balance between jet thrust and the ram pressure of the external medium, characterised by the ratio

$$
\eta=\frac{L}{R^{2} c^{3} \rho}
$$

where $L$ is the jet power, $R$ is the characteristic dimension of the jet, $c$ is the speed of light and $\rho$ is the density of the external medium. Since the properties of the jet (power and opening angle) are 
set at the base, where lengths and timescales scale with the black hole mass, then $L \propto M$ and $R \propto M$. For scale invariance of the jet dynamics, we then require $\rho \propto M^{-1}$. While supermassive black holes are a factor $10^{6}-10^{9}$ times more massive than their stellar mass cousins, then given typical IGM densities within clusters of $10^{-5}-10^{-2} \mathrm{~cm}^{-3}$, then for scale invariance, the ISM densities around XRB jets would need to be $10^{1}-10^{7} \mathrm{~cm}^{-3}$, whereas in practice, they span the range $10^{-3}-10^{4} \mathrm{~cm}^{-3}$. Only in the densest regions of the Galaxy can we expect scale invariance. Otherwise, XRB jets are located in dynamically underdense environments when compared to AGN.

\subsection{Observable characteristics}

In such dense regions, we can search for the signatures of jet-ISM interactions. These take three forms; hotspots, bow shocks and lobes. The working surfaces where the jets impact the ISM may be observed as hotspots, where shock-accelerated electrons radiate non-thermal synchrotron emission. Over time, the jets will inflate a bubble around the central source, filled with old synchrotron-emitting plasma, and surrounded by a shell of swept-up ISM, which emits bremsstrahlung radiation, and, in the case of a radiative shock, also significant line emission. Applying a self-similar luminosity-driven expansion model to such a source gives predictions for the surface brightness of the expected emission from shells and lobes [5]. The expected radio surface brightness of the synchrotron emission from the lobes is of order

$$
S_{v} \approx 50 \frac{\mu \mathrm{Jy}}{\operatorname{arcsec}^{2}}\left(\frac{P}{10^{36} \mathrm{erg} \mathrm{s}^{-1}}\right)^{0.9}\left(\frac{t}{10^{5} \mathrm{y}}\right)^{-0.8}\left(\frac{n}{1 \mathrm{~cm}^{-3}}\right)^{0.85}\left(\frac{v}{5 \mathrm{GHz}}\right)^{-0.5} f_{\mathrm{p}, B},
$$

where $P$ is the jet power, $t$ is the age of the source, $n$ is the density of the medium, $v$ is the observing frequency, and $f_{\mathrm{p}, B}$ is a correction factor to account for the energy in magnetic fields and protons, for sources out of equipartition. Powerful, young sources in dense environments are most easily detected, and we need to observe at low frequencies. While the sensitivity of current radio facilities is not sufficient to detect such structures in most cases [6], upcoming low-frequency arrays such as LOFAR, MWA and LWA will provide significant advances in our capabilities. For the free-free emission from shocked ISM shells, the maximum expected surface brightness is

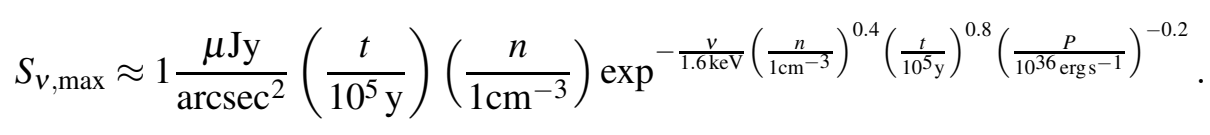

Once again, it is easiest to detect powerful sources in dense environments.

For the case of hotspots, predictions have been made for the spectral signature of jets from XRB systems interacting with a dense molecular cloud [7]. If the jets contain a significant component of relativistic protons, they will produce high-energy emission ( $\mathrm{GeV}$ and even $\mathrm{TeV}$ energies) via pion decay, as well as lower-frequency emission from synchrotron, bremsstrahlung and inverse Compton emission from secondary leptons. Molecular clouds at distances of 10-100 pc were considered, corresponding to angular separations of several arcminutes at typical X-ray binary distances. However, as we will see in Section 3.3, most detected hotspots have been closer to the central binary system, at angular separations of a few arcseconds. Closer interaction sites would enhance the predicted levels of emission. 


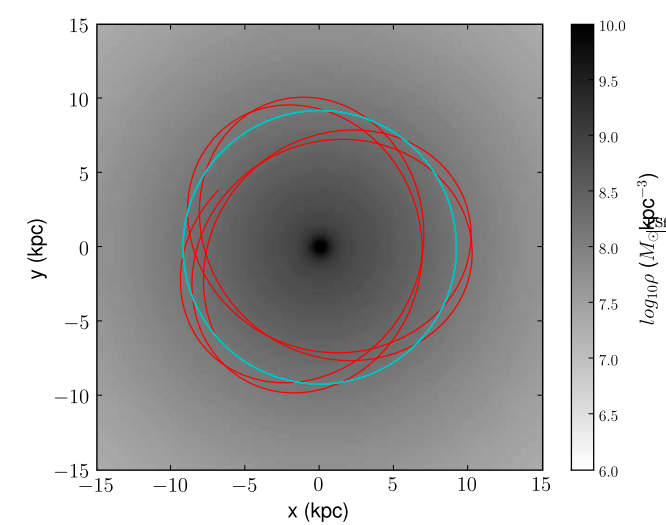

(a) Greyscale shows the density in the plane of the Galaxy, red trace shows the calculated orbit of the black hole X-ray binary V404 Cyg in the Galactic potential for the last $1 \mathrm{Gyr}$, blue trace shows the corresponding orbit of the LSR of 1 Gyr ago.

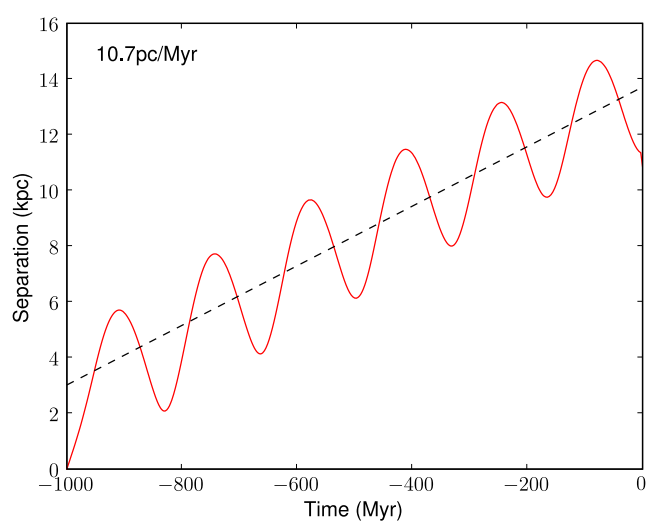

(b) Red trace shows the distance between the positions of V404 Cyg and its LSR of $1 \mathrm{Gyr}$ ago. The system moves with an average space velocity of $10.7 \mathrm{pc} \mathrm{Myr}^{-1}$ (dotted line) with respect to the LSR.

Figure 1: The motion of the black hole X-ray binary V404 Cyg in the potential of the Galaxy [9]. The source moves at an average of $10.7 \mathrm{pc} \mathrm{Myr}^{-1}$ with respect to its LSR.

\subsection{Radio trails}

Since XRBs are moving through the Galactic potential, the jets are propagating through a moving medium (Fig. 1). As the jets propagate through the ISM, they slow to sub-relativistic velocities, and eventually the dynamical pressure of the X-ray binary motion through the ISM becomes important, slowing the advance speed of the working surface until it becomes stationary in the X-ray binary frame. Plasma released at the working surface is swept back with the ISM creating a trail of waste plasma, which expands adiabatically [3]. Such plasma trails can extend for up to $1 \mathrm{kpc}$ in length, and are preferentially found in old, low-power systems with a high space velocity, located in a dense environment. Their predicted radio flux densities are on the order of a few microJy $\operatorname{arcsec}^{-2}$ (scaling with jet power, Mach number, ISM pressure, and X-ray binary space velocity). They will be destroyed by shearing forces from the differential rotation of the Galaxy and dynamical instabilities mixing the plasma with the ISM, and by buoyancy effects moving them out into the Galactic halo. Table 1 lists the peculiar velocities of the X-ray binaries with measured threedimensional space velocities. The best candidates in which to observe plasma trails are LMXBs with a high space velocity, such as XTE J 1118+480, GRO J 1655-40, or Sco X-1. The candidate jet-blown lobe to the southwest of Cygnus X-3 [8] was suggested to be a candidate radio trail [3], but a recent measurement of the proper motion of the system (Sakari et al., in prep.) showed the source to be moving to the southwest, ruling out the identification of the extended radio emission as a plasma trail.

\subsection{Feedback effects}

The release of plasma by X-ray binary jets into the surrounding medium can have a significant 


\begin{tabular}{|c|c|c|c|c|}
\hline Source & $v_{\text {pec }}\left(\mathrm{km} \mathrm{s}^{-1}\right)$ & Class & Nebula? & References \\
\hline GRS $1915+105$ & $38 \pm 67$ & LMXB & & {$[10,11]$} \\
\hline V404 Cyg & $67 \pm 78$ & LMXB & & \\
\hline XTE J $1118+480$ & $160 \pm 25$ & LMXB & & [12] \\
\hline GRO J 1655-40 & $115 \pm 21$ & LMXB & & [13] \\
\hline Cygnus X-1 & $34 \pm 27$ & HMXB & Y & [14] \\
\hline Cygnus X-3 & $>40$ & HMXB & & \\
\hline SS 433 & $32 \pm 3$ & HMXB & $\mathrm{Y}$ & [15] \\
\hline Sco X-1 & $235 \pm 15$ & LMXB & & [16] \\
\hline Cir X-1 & $184 \pm 40$ & HMXB? & Y & [17] \\
\hline $\mathrm{LSI}+61^{\circ} 303$ & $21 \pm 1$ & HMXB & & [18] \\
\hline LS 5039 & $120 \pm 24$ & HMXB & & [19] \\
\hline
\end{tabular}

Table 1: Derived peculiar velocities, relative to the LSR, of X-ray binary systems known to have relativistic jets. Measured proper motions, radial velocities, positions and distances of the systems have been converted to heliocentric velocity components $U, V$ and $W$ using the transformations of [20]. The peculiar velocity, $v_{\text {pec }}$, i.e., the difference in measured velocity and that expected for a system following the Galactic rotation, was calculated, assuming a flat rotation curve of circular velocity $220 \mathrm{~km} \mathrm{~s}^{-1}$ and a Galactocentric distance of $8 \mathrm{kpc}$.

feedback effect. The energy output of jets, estimated to be of order $1 \%$ of the time-averaged luminosity of supernovae [21], has an effect in heating the ISM, generating turbulence, and acting as a source of Galactic cosmic rays [22]. Furthermore, the plasma released into the ISM (whose volume over the lifetime of the galaxy is comparable to that of the entire Galactic disc) also provides a seed magnetic field, which can be amplified via the dynamo effect of Galactic rotation to produce the observed Galactic field [3].

\section{Observed jet-ISM interactions}

\subsection{Jet-blown lobes}

We know of only a handful of systems where we observe the radio-emitting lobes inflated by the action of the X-ray binary jets. The first to be discovered, and probably the most well-studied, is the W 50 nebula, shaped by the interaction of the precessing jets of SS 433 with the central supernova remnant. The system lies $\sim 2^{\circ}$ below the Galactic Plane (Fig. 2), so the precessing jets are expanding into a density gradient, which accounts for the asymmetry between the less extended, brighter western lobe, and the larger, fainter eastern lobe. Simulations have shown that a jet interacting with a pre-existing supernova remnant can reproduce the observed morphology of the nebula [23, 24]. A detailed inspection of the H I emission in the vicinity of the nebula [15] showed further evidence for the impact of the jets on their surroundings; an expanding shell of radius $40 \mathrm{pc}$ in direct alignment with the jet axis, beyond the eastern end of the nebula. With a mass of $8 \pm 3 M_{\odot}$ and an energy of $3 \pm 1.5 \times 10^{49} \mathrm{erg}$, it is thought to have been inflated by the jets at some more active stage in the past. 


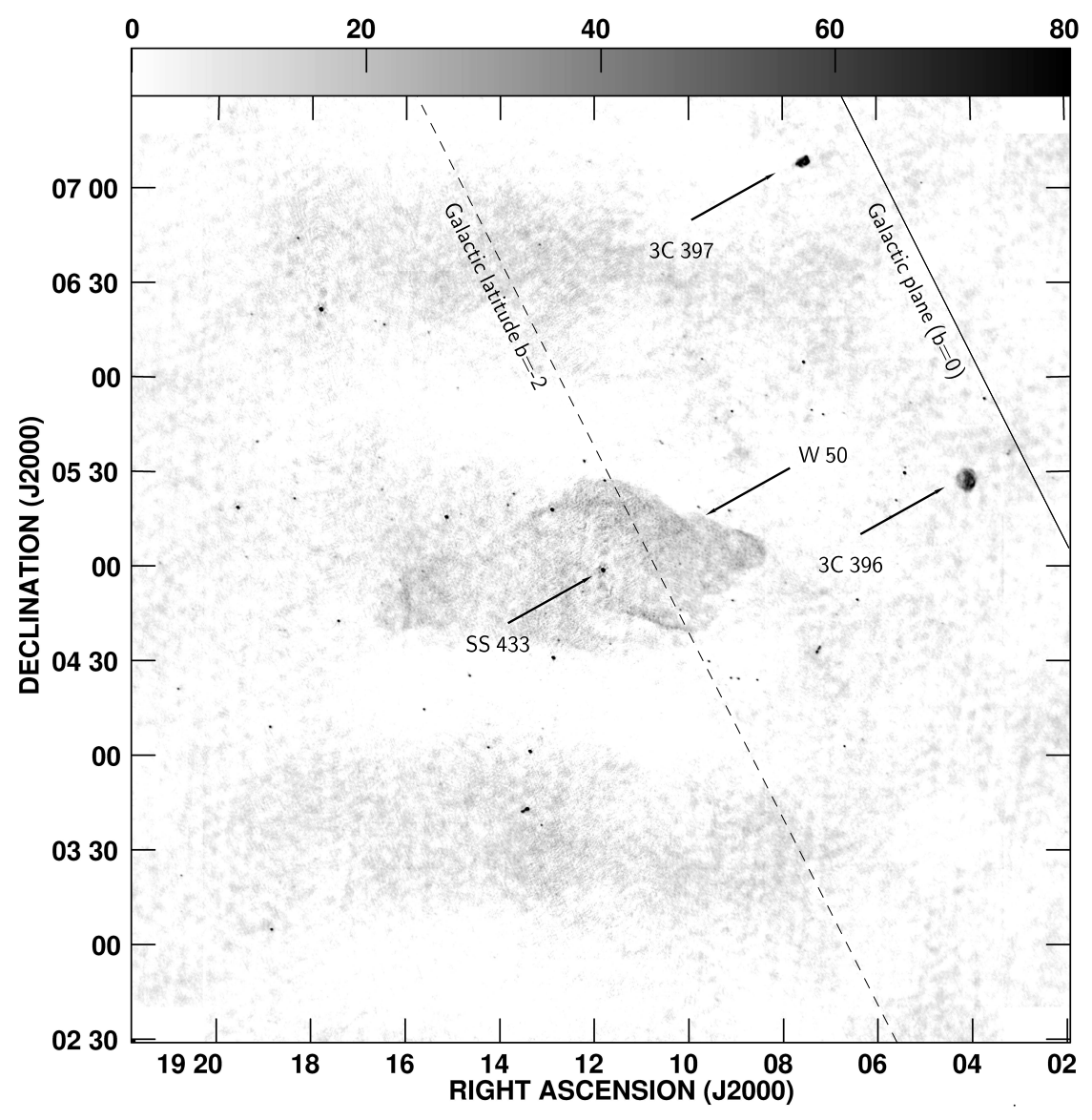

Figure 2: SS 433, W 50, and the surrounding region of the Galactic Plane.

The field of microquasars was opened up in 1992 by the discovery of radio lobes associated with the two Galactic Centre sources 1E 1740.7-2942 [25] and GRS 1758-258 [26]. In both cases, time-variable cores were found to be at the centre of persistent, steep-spectrum radio lobes, which terminated in bright hotspots, reminiscent of the morphology of AGN. It is likely that the location of the sources in the Galactic Centre region provides a sufficiently dense environment to create detectable radio-emitting lobes. Further radio observations of GRS 1758-258 suggested that the minimum pressure in the lobes was comparable to that in Galactic molecular clouds, reinforcing the hypothesis of a dense environment. The lack of evidence for synchrotron ageing in the northern lobe suggests that the age of the lobes is $<1 \mathrm{Myr}$ [27], so they are young sources.

The final known case of jet-blown lobes is Circinus X-1. This neutron-star X-ray binary lies at the centre of a radio nebula [28]. The arcsecond-scale jets, the most relativistic known in an X-ray binary system [29], are oriented very close to the line of sight, and are believed to have inflated the surrounding nebula, such that we are observing the central source through the jet-inflated lobes [30]. Applying a self-similar model originally developed for AGN jets [31] allowed estimates of the jet power $\left(>10^{35} \mathrm{erg} \mathrm{s}^{-1}\right)$ and age $\left(2 \times 10^{4} \mathrm{y}\right)$ of the system [30]. This is one of the best constraints to date for the power of a neutron star jet. 


\subsection{Shells}

The only confirmed case to date where a shell of shocked, compressed ISM has been detected around an X-ray binary is Cygnus X-1 [32]. A shell of emission aligned with the milliarcsecondscale jet seen in this source [33] was detected in radio and $\mathrm{H} \alpha$ emission. The density enhancement as the northern jet encounters the tail of a nearby $\mathrm{H}$ II region allows the shell to be detected, whereas no lobe is seen corresponding to the southern counterjet. The shell can be used as a calorimeter to constrain the product of the jet power and jet lifetime. Optical line observations [34] confirmed that the shell was shock-excited, and constrained the velocity of the shock to be $\geq 100 \mathrm{~km} \mathrm{~s}^{-1}$, the jet lifetime to be $17-63 \mathrm{kyr}$, and the time-averaged jet power to fall in the range (4-14) $\times 10^{36} \mathrm{erg} \mathrm{s}^{-1}$. Once again, we see evidence of jet-ISM interaction due to the combination of a young, powerful jet and a dense environment.

\subsection{Hotspots}

As well as inflating a radio nebula (Section 3.1), the jets of Cir X-1 show hotspots, the working surfaces where they interact with the surroundings. Flaring in the core was observed to be closely followed by a brightening of the hotspots, implying an unseen, underlying, highly-relativistic flow with a minimum Lorentz factor of $\Gamma>15$ [29]. Evidence for such an underlying relativistic flow has also been seen in another neutron star system, Sco X-1 [35, 36]. Pairs of emitting lobes were observed to move away from the stationary core at speeds of $0.45 c$, and were inferred to have been energised by an underlying flow with a velocity $>0.95 c$. The lobes were identified with the working surfaces of the energy flow on the surroundings. Electrons diffused away from the hotspots at $0.57 c$, and either adiabatic expansion or radiative losses is believed to have prevented the formation of large, extended lobes behind the working surface. Interestingly, both of these sources are neutron star X-ray binaries.

A third source in which arcsecond-scale radio lobes have been detected is the black hole system GX 339-4 [37]. After an outburst in 2002 May, large-scale extended jets were seen in 2003 January and May, at angular separations of 5.5 and 6.9 arcsec respectively. Although the source distance is uncertain, this implies a projected distance of at least $0.14 \mathrm{pc}$ and a minimum velocity of $0.5 c$. The radio emission was identified as the interaction of the jets with ambient material.

Similar large-scale emission seen months to years after an outburst has also been detected in the X-ray and radio bands in the sources XTE J 1550-564 [38, 39] and H 1743-322 [40]. X-ray and radio-emitting knots were observed to appear at distances of a few tenths of a parsec from the central source. The spectral energy distribution was consistent in both cases with an optically-thin power law synchrotron spectrum from the radio to the X-ray band. In XTE J 1550-564, the radio morphology of the western jet knot was seen to be extended both along and perpendicular to the jet axis, forming a bowshock-like structure [41]. The observed deceleration of the knots (Section 3.4) confirms their identification as the hotspots where the jets interact with the ISM.

Searches for hotspots around sources such as GRS 1915+105 [42, 6] and Cygnus X-3 [43] identified twin radio sources equidistant from the central source and in alignment with the smallscale jet axis, as potential hotspots in both these sources. However, the Cygnus X-3 hotspot candidates are now believed to be foreground sources or background AGN [44], and the evidence for an 


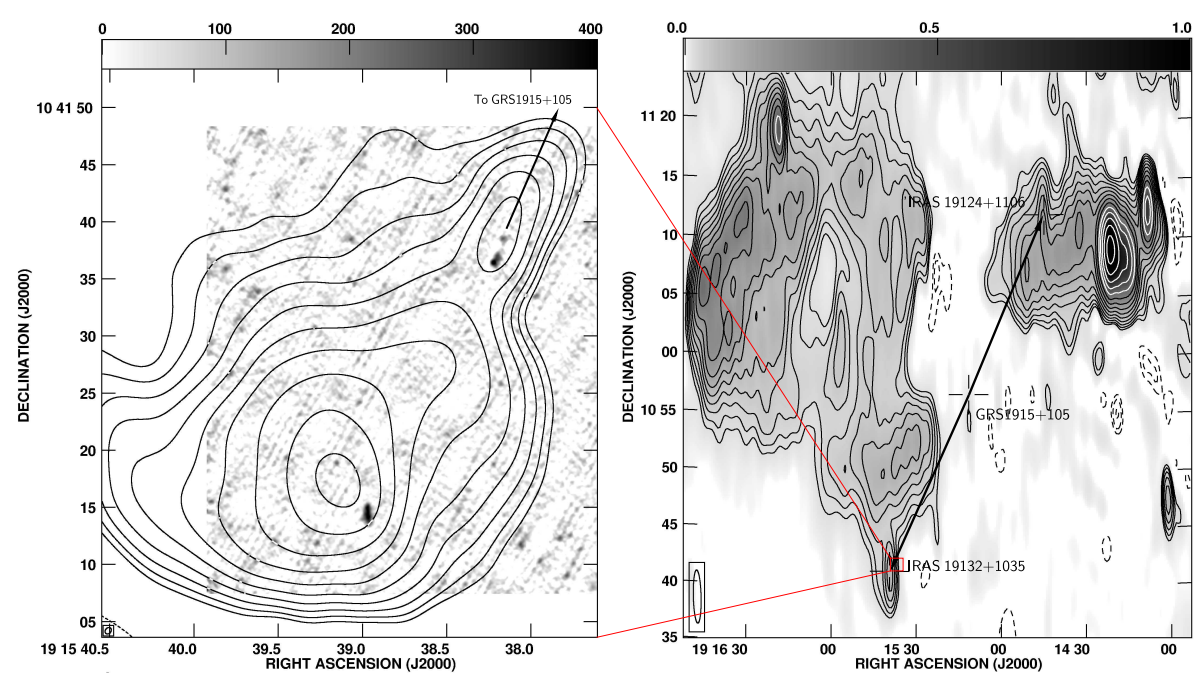

Figure 3: The environment around GRS 1915+105. Right: 92-cm WSRT image of the field, showing the $\mathrm{X}$-ray binary and the two proposed hotspots. No evidence for synchrotron lobes linking the sources back to GRS $1915+105$ is observed. Left: $1.4-\mathrm{GHz}$ VLA contour map superposed on MERLIN grayscale image of the southeastern source, IRAS 19132+1035. We see no definitive evidence for a bowshock structure indicative of jet-ISM interactions. Figure adapted from [46].

association between the two sources around GRS 1915+105 (Fig. 3) and the central binary system is inconclusive at best $[45,46]$.

\subsection{Decelerating jets}

In the case of XTE J 1550-564, gradual deceleration of the X-ray emitting jet knots was observed [39]. For a single impulsive ejection event, the jet knot sweeps up the ambient material as it moves outwards, and is slowed down once it has swept up a fraction $1 / \Gamma$ of its own mass [4], where $\Gamma$ is the bulk Lorentz factor of the jet. Thus it will appear to decelerate after a distance

$$
d_{\text {slow }} \sim 10^{16}\left[\left(\frac{E}{10^{44} \mathrm{erg}}\right)\left(\frac{\Gamma}{5}\right)^{-2}\left(\frac{\theta}{5^{\circ}}\right)^{-1}\left(\frac{n}{1 \mathrm{~cm}^{-3}}\right)^{-1}\right]^{1 / 3} \mathrm{~cm},
$$

where $E$ is the kinetic energy of the ejecta, $n$ the density of the surrounding medium, and $\theta$ the opening angle of the jet [4].

Deceleration was also seen in the 1998 outburst of XTE J 1748-288 [46]. The measured proper motion over the first $20 \mathrm{~d}$ of the outburst suggested that the ejecta must have decelerated between the detected X-ray flare and the first radio detection, within 200 mas of the central binary. The jet was then seen to speed up, before stalling a second time at an angular separation of $\sim 1$ arcsec. This is suggestive of complex interactions with the environment, and analysis is still ongoing.

The discrepancy in the proper motions of the jets of GRS 1915+105, between those measured on milliarcsecond scales with the VLBA and MERLIN (23 mas $\mathrm{d}^{-1}$; [47]), and on arcsecond scales with the VLA (17 mas $\mathrm{d}^{-1}$; [48]), was indicative of possible deceleration on intermediate scales. However, an observing campaign using all three instruments, coupled with a reanalysis of all the archival data [49] showed no evidence for any deceleration, unless it occurred within 70 mas of the core. It is more likely that the jet speed in this system is variable. 


\subsection{Jet deflection and destruction}

High-mass X-ray binary systems tend to have strong winds from the donor star, which will certainly affect the immediate environment of the jets, particularly close to the central binary system where the density of the wind is highest. Such winds may well be inhomogeneous or clumpy, and the interaction of the jets with the wind will produce strong shocks, and may even lead to the disruption and deflection of the jets close to the central binary system, preventing the formation of large-scale jets for jet luminosities $<10^{36} \mathrm{erg} \mathrm{s}^{-1}$ [50].

A prime example of a case where jets are believed to have been disrupted by a dense circumstellar medium is the 1998 outburst of CI Cam [51]. VLBI imaging showed the development of an expanding, clumpy, synchrotron-emitting structure, suggestive of a decelerating shock moving through dense material from the stellar wind. Another example is provided by the case of Cygnus X-3. VLBI imaging of the resolved jets in this system [52, 53, 54] shows a variety of different jet morphologies, including one-sided and two-sided jets, with kinks and regions of enhanced polarization in areas where the jet is believed to be interacting with its environment. The structures have been difficult to interpret in some cases [54], but a recent determination of the proper motion (Sakari et al., in prep.) allows the determination of the core position at any given epoch. Interpreting the VLBI images using the new core position shows that while the one-sided jets went to the south in the 1997 outburst [52], they went to the north in the 2006 outburst [54]. Such an apparent $180^{\circ}$ deflection can be explained if the jets are aligned very close to the line of sight, with projection effects causing large apparent deflections. However, some deviation of the jet propagation direction is still required, so in the absence of genuine precession (a precession cone with a wide opening angle is unlikely, given the north-south orientation seen in all epochs), jet deflection by the stellar wind is a plausible explanation.

Two other high-mass X-ray binary systems in which even the existence of a jet is debated, LSI $+61^{\circ} 303$ and LS 5039, are notable for their observed $\mathrm{TeV}$ emission. Both show resolved radio emission, with a position angle which varies with time $[18,55]$. The nature of the radio emission is still under debate. The precessing, cometary morphology of the radio emission in LSI $+61^{\circ} 303$ led [18] to deduce that the radio emission arose from a pulsar wind interacting with the dense wind of the companion Be star. Similar changing morphology has recently been seen in orbital phase-resolved radio imaging of LS 5039 [19]. However, recent theoretical work claimed that for a pulsar wind sufficiently powerful to produce the observed gamma-ray luminosity in LSI $+61^{\circ} 303$, the predicted radio morphology would differ from that observed, being less well collimated, and centred on the Be star rather than the pulsar [63]. If, instead, the radio morphology is explained by an accretion-ejection scenario, the changing morphology could be attributed to disruption of the jet by the wind of the donor star as the compact object approaches periastron [50].

SS 433 is one case of a high-mass system where the stellar wind does not appear to disrupt the jets. The mass donor is believed to be an A-type supergiant [56], accreting at a very high rate. As well as the wind from the donor star, radio observations have shown evidence for a radiation pressure-driven disc wind in the equatorial plane [57, 58], whose estimated mass-loss rate of $\sim 10^{-4} M_{\odot} \mathrm{yr}^{-1}$ exceeds that in the jets by two orders of magnitude. Despite the complicated, dense environment, the precessing jets in SS 433 are ever-present, and follow the predictions of the kinematic model $[59,60,61]$ extremely well, with no evidence for jet deflection or destruction. 


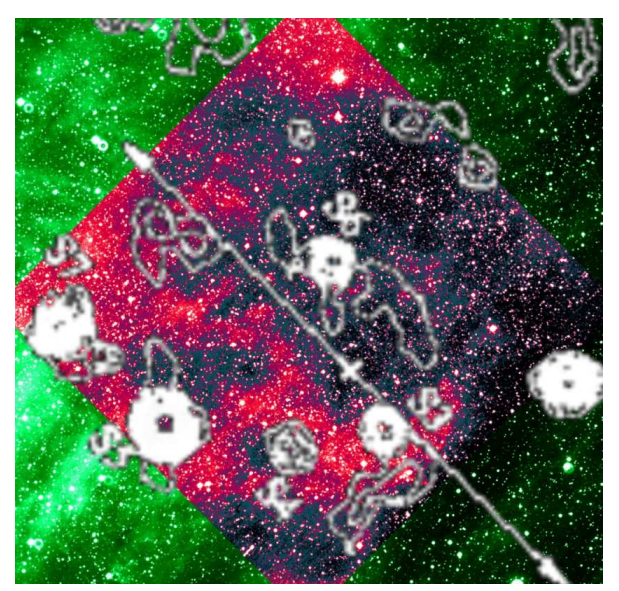

Figure 4: The surroundings of GRO J 1655-40. Green shows $\mathrm{H} \alpha$ emission, red shows S II emission, white shows $1.4-\mathrm{GHz}$ radio contours [65]. The cross marks the position of the X-ray binary and the arrows denote the orientation of the relativistic jets [66].

Theoretical modelling [62] has shown that jets surrounded by an outflowing wind can in fact be more stable than those surrounded by a stationary or inflowing atmosphere. It is possible that the disc wind, with the same foot point as the jet, could help collimate the jet, whereas stellar winds, through which the jet must propagate more laterally, are more likely to cause disruption.

\section{Future prospects}

The detection of optical line emission from the shell surrounding Cygnus X-1 [32,34] suggests that this could be an alternative search technique to deep, wide-field radio imaging. A preliminary $\mathrm{H} \alpha$ survey of the fields surrounding $30 \mathrm{X}$-ray binaries was carried out [64], yielding three potential candidates, GRS 1009-45, LMC X-1, and the best case, GRO J 1655-40. In the latter case, an $\mathrm{H} \alpha$ shell surrounding the X-ray binary was also visible in [S II] emission lines, a tracer of shockexcited gas. This shell is also observed in 843 and $1400 \mathrm{MHz}$ radio emission (Fig. 4). Follow-up observations have been taken to ascertain whether this is a second example of a shock-compressed shell of ISM inflated by X-ray binary jets.

In Section 2, we found that we would expect radio lobes inflated by the jets of X-ray binaries to be faint, but brighter at low frequencies. Furthermore, arising from lower-energy electrons, the lower-frequency emission will be longer-lived than that at $\mathrm{GHz}$ frequencies. The next generation of radio facilities coming online [67] will significantly increase the likelihood of detecting such structures. LOFAR, the MWA and the LWA will be ideal for observing low surface brightness extended emission. Furthermore, one of the operating modes of LOFAR will be as a radio all-sky monitor [68]. With a field of view of several degrees, and sub-millijansky sensitivity, it will be ideally placed to detect synchrotron emission from radio rebrightening at sites of jet-ISM interaction as seen in GX 339-4 or XTE J 1550-564 (Section 3.3), and to follow such emission as it propagates outwards over the course of several months to years. The EVLA and eMERLIN, on the other hand, will be better suited to probing faint, compact structures, and resolving the interaction regions initially detected by wider-field monitoring instruments such as LOFAR. 
To date, no plasma trails (Section 2.2) have yet been detected from an X-ray binary system. The predicted low surface brightnesses imply that the next generation of facilities will be necessary to observe them. Again, they are predicted [3] to be brighter at lower frequencies, making them another possible candidate for LOFAR or the MWA. As the space velocities of more X-ray binary systems are measured, the list of likely candidates grows. The three highest-velocity objects, GRO J 1655-40, Sco X-1, and XTE J 1118+480 are the best candidates for a targetted search (Table 1). The latter two systems, however, lie $23^{\circ}$ and $62^{\circ}$, respectively, off the Galactic plane, implying that they inhabit lower-density, lower-pressure halo environments where the predicted surface brightness of the trails would be lower.

To date, only three X-ray binary systems have been detected in the very high energy $(\mathrm{GeV}$ and $\mathrm{TeV}$ ) regime $[69,70,71,72]$. With the new generation of instruments such as MAGIC II, HESS II, CTA and Fermi, more detections may well follow. Comparison of the observed spectral energy distributions with theoretical models may establish whether we see the signatures of jets interacting with the ISM. This would also help to determine the hadronic or leptonic nature of X-ray binary jets.

\section{Conclusions}

Evidence is emerging in a number of systems for the interactions of the jets of $\mathrm{X}$-ray binaries with their surroundings. This is seen in three main forms: synchrotron-emitting radio lobes; sweptup shells of collisionally-ionised ISM emitting bremsstrahlung radiation; and hotspots, the working surfaces where the jets impact on the ISM. Searches in the radio, X-ray and optical bands have all made contributions to the detection of such interactions. Such observations can provide important information about the power and lifetimes of X-ray binary jets, and the density of the interstellar medium.

While the surrounding medium can affect the jets by confining, smothering, or deflecting them, the jets also have a significant feedback effect on the ISM. They inject large amounts of energy, and momentum into the ambient medium, and can seed the surroundings with magnetic fields. They inflate large volumes of relativistic plasma, and contribute a few percent of the Galactic cosmic ray population.

With the next generation of radio facilities, and using deeper optical line surveys, we expect to be able to detect more such structures, opening up this field further and providing extra feedback for theoretical models.

\section{Acknowledgments}

I would like to thank all my collaborators, particularly Katherine Blundell, Vivek Dhawan, Rob Fender, Christian Kaiser, Anna Kapińska, Dave Russell, Charli Sakari and Valeriu Tudose. The National Radio Astronomy Observatory is operated by Associated Universities, Inc., under cooperative agreement with the National Science Foundation. 


\section{References}

[1] A. Merloni, S. Heinz, T. di Matteo, A Fundamental Plane of black hole activity, MNRAS 03 (345) 1057 [arXiv: astro-ph/0305261].

[2] H. Falcke, E. Körding, S. Markoff, A scheme to unify low-power accreting black holes. Jet-dominated accretion flows and the radio/X-ray correlation, MNRAS 04 (414) 895

[arXiv:astro-ph/0305335].

[3] S. Heinz, H.J. Grimm, R.A. Sunyaev, R.P. Fender, Blazing trails: Microquasars as head-tail sources and the seeding of magnetized plasma into the ISM, ApJ $\mathbf{0 8}$ [arXiv:0808.1927].

[4] S. Heinz, Radio lobe dynamics and the environment of microquasars, A\&A 02 (388) L40 [arXiv:astro-ph/0203295].

[5] S. Heinz, How Deep is Your Lobe? Microquasars and Their Impact on the ISM, in proceedings of $A$ Population Explosion: The Nature \& Evolution of X-ray Binaries in Diverse Environments, $\mathbf{0 8 b}$ AIP Conf. Ser., (1010) 40

[6] C.R. Kaiser, K.F. Gunn, C. Brocksopp, J.L. Sokoloski, Revision of the Properties of the GRS 1915+105 Jets: Clues from the Large-Scale Structure, ApJ 04 (612) 332 [arXiv:astro-ph/0405206].

[7] V. Bosch-Ramon, F.A. Aharonian, J.M. Paredes, Electromagnetic radiation initiated by hadronic jets from microquasars in the ISM, A\&A 05 (432) 609 [arXiv: astro-ph/ 0411508 ].

[8] J.R. Sánchez-Sutil, J. Martí, J.A. Combi, P. Luque-Escamilla, A.J. Muñoz-Arjonilla, J.M. Paredes, $\mathrm{G}$. Pooley, Faint arc-minute extended radio emission around Cygnus $X-3, A \& A \mathbf{0 8}$ (479) 523 [0801.1205].

[9] K.V. Johnston, D.N. Spergel, L. Hernquist, The Disruption of the Sagittarius Dwarf Galaxy, ApJ 95 (451) 598 [arXiv:astro-ph/9502005].

[10] J. Greiner, J.G. Cuby, M.J. McCaughrean, An unusually massive stellar black hole in the Galaxy, Nature 01 (414) 522 [arXiv: astro-ph/0111538].

[11] V. Dhawan, I.F. Mirabel, M. Ribó, I. Rodrigues, Kinematics of Black Hole X-Ray Binary GRS 1915+105, ApJ 07 (668) 430 [arXiv: 0705.1800$].$

[12] I.F. Mirabel, V. Dhawan, R.P. Mignani, I. Rodrigues, F. Guglielmetti, A high-velocity black hole on a Galactic-halo orbit in the solar neighbourhood, Nature 01 (413) 139 [arXiv:astro-ph/0109098].

[13] I.F. Mirabel, R. Mignani, I. Rodrigues, J.A. Combi, L.F. Rodríguez, F. Guglielmetti, The runaway black hole GRO J1655-40 02 (395) 595 [arXiv: astro-ph/ 021144 5].

[14] I.F. Mirabel, I. Rodrigues, Formation of a Black Hole in the Dark, Science 03 (300) 1119 [arXiv:astro-ph/0305205].

[15] F.J. Lockman, K.M. Blundell, W.M. Goss, The distance to SS433/W50 and its interaction with the interstellar medium, MNRAS 07 (381) 881 [arXiv: 0707.0506 ].

[16] C.F. Bradshaw, E.B. Fomalont, B.J. Geldzahler, High-Resolution Parallax Measurements of Scorpius $X-1, A p J(512) \mathrm{L} 121$.

[17] R.P. Mignani, A. De Luca, P.A. Caraveo, I.F. Mirabel, HST observations rule out the association between Cir X-1 and SNR G321.9-0.3, A\&A 02 (386) 487 [arXiv: astro-ph/ 0202268 ]. 
[18] V. Dhawan, A. Mioduszewski, M. Rupen, LS I +61 303 is a Be-Pulsar binary, not a Microquasar, in proceedings of VI Microquasar Workshop: Microquasars and Beyond, PoS ( MQW6) 052.

[19] J. Moldon, Exploring the powering source of the TeV X-ray binary LS 5039, these proceedings, VII Microquasar Workshop: Microquasars and Beyond, POS ( MQW7) ? ? .

[20] D.R.H. Johnson, D.R. Soderblom, Calculating galactic space velocities and their uncertainties, with an application to the Ursa Major group, AJ 87 (93) 864.

[21] R.P. Fender, T.J. Maccarone, Z. van Kesteren, Energization of interstellar media and cosmic ray production by jets from X-ray binaries, MNRAS 05 (360) 1085 [arXiv: astro-ph/ 0504205 ].

[22] S. Heinz, R. Sunyaev, Cosmic rays from microquasars: A narrow component to the CR spectrum?, A\&A 02 (390) 751 [arXiv:astro-ph/ 0204183 ].

[23] P.F. Velázquez, A.C. Raga, A numerical simulation of the W 50-SS 433 system, A\&A 00 (362) 780.

[24] J. Zavala, P.F. Velázquez, A.H. Cerqueira, G.M. Dubner, Three-dimensional hydrodynamical simulations of the large-scale structure of W50-SS433, MNRAS 08 (387) 839.

[25] I.F. Mirabel, L.F. Rodriguez, B. Cordier, J. Paul, F. Lebrun, A double-sided radio jet from the compact Galactic Centre annihilator 1E140.7 - 2942, Nature 92 (358) 215.

[26] L.F. Rodriguez, I.F. Mirabel, J. Marti, The radio counterpart of the hard X-ray source GRS 1758-258, ApJ 92 (401) L15.

[27] M.J. Hardcastle, Low-frequency constraints on the spectra of the lobes of the microquasar GRS 1758 258, A\&A 05 (434) 35 [arXiv: astro-ph/ 0501060 ].

[28] R.F. Haynes, M.M. Komesaroff, D.L. Jauncey, J.L. Caswell, D.K. Milne, M.J. Kesteven, K.J. Wellington, R.A. Preston, A radio nebula associated with Circinus X-1, Nature 86 (324) 233.

[29] R. Fender, K. Wu, H. Johnston, T. Tzioumis, P. Jonker, R. Spencer, M. van der Klis, An ultra-relativistic outflow from a neutron star accreting gas from a companion, Nature 04 (427) 222 [arXiv:astro-ph/0401290].

[30] V. Tudose, R.P. Fender, C.R. Kaiser, A.K. Tzioumis, M. van der Klis, R.E. Spencer, The large-scale jet-powered radio nebula of Circinus X-1, MNRAS 06 (372) 417 [arXiv : a stro-ph / 0607612 ].

[31] C.R. Kaiser, P. Alexander, A self-similar model for extragalactic radio sources, MNRAS 97 (286) 215.

[32] E. Gallo, R. Fender, C. Kaiser, D. Russell, R. Morganti, T. Oosterloo, S. Heinz, A dark jet dominates the power output of the stellar black hole Cygnus X-1, Nature 05 (436) 819 [arXiv:astro-ph/0508228].

[33] A.M. Stirling, R.E. Spencer, C.J. de la Force, M.A. Garrett, R.P. Fender, R.N. Ogley, A relativistic jet from Cygnus X-1 in the low/hard X-ray state, MNRAS 01 (327) 1273 [arXiv:astro-ph/0107192].

[34] D.M. Russell, R.P. Fender, E. Gallo, C.R. Kaiser, The jet-powered optical nebula of Cygnus X-1, MNRAS 07 (376) 1341 [arXiv:astro-ph/ 0701645 ].

[35] E.B. Fomalont, B.J. Geldzahler, C.F. Bradshaw, Scorpius X-1: Energy Transfer from the Core to the Radio Lobes, ApJ 01 (553) L27 [arXiv: astro-ph/ 0104325$].$

[36] E.B. Fomalont, B.J. Geldzahler, C.F. Bradshaw, Scorpius X-1: The Evolution and Nature of the Twin Compact Radio Lobes, ApJ 01 (558) 283 [arXiv: astro-ph/ 0104372 ]. 
[37] E. Gallo, S. Corbel, R.P. Fender, T.J. Maccarone, A.K. Tzioumis, A transient large-scale relativistic radio jet from GX 339-4, MNRAS 04 (347) L52 [arXiv : astro-ph/0311452].

[38] S. Corbel, R.P. Fender, A.K. Tzioumis, J.A. Tomsick, J.A. Orosz, J.M. Miller, R. Wijnands, P. Kaaret, Large-Scale, Decelerating, Relativistic X-ray Jets from the Microquasar XTE J1550-564, Science 02 (298) 196 [arXiv:astro-ph/0210224].

[39] P. Kaaret, S. Corbel, J.A. Tomsick, R. Fender, J.M. Miller, J.A. Orosz, A.K. Tzioumis, R. Wijnands, $X$-Ray Emission from the Jets of XTE J1550-564, ApJ 03 (582) 945 [arXiv:astro-ph/0210401].

[40] S. Corbel, P. Kaaret, R.P. Fender, A.K. Tzioumis, J.A. Orosz, Discovery of X-Ray Jets in the Microquasar H1743-322, ApJ 05 (632) 504 [arXiv: astro-ph/ 0505526 ].

[41] S. Corbel, Long-term evolution of the X-ray jets in XTE J 1550-564, these proceedings, VII Microquasar Workshop: Microquasars and Beyond, POS ( MQW7) 049.

[42] L.F. Rodriguez, I.F. Mirabel, The surroundings of the superluminal source GRS 1915+105, A\&A 98 (340) L47 [arXiv:astro-ph/9811250].

[43] J. Martí, D. Pérez-Ramírez, J.L. Garrido, P. Luque-Escamilla, J.M. Paredes, Possible hot spots excited by the relativistic jets of Cygnus X-3, A\&A 05 (439) 279 [arXiv: astro-ph/ 0505433 ].

[44] J. Martí, D. Pérez-Ramírez, P. Luque-Escamilla, J.L. Garrido, J.M. Paredes, A.J. Muñ oz-Arjonilla, J.R. Sánchez-Sutil, A radio and infrared exploration around Cygnus X-3, A\&A 06 (451) 1037.

[45] S. Chaty, L.F. Rodríguez, I.F. Mirabel, T.R. Geballe, Y. Fuchs, A. Claret, C.J. Cesarsky, D. Cesarsky, A search for possible interactions between ejections from GRS $1915+105$ and the surrounding interstellar medium, A\&A 01 (366) 1035 [arXiv : astro-ph/ 0011297 ].

[46] J. Miller-Jones et al., Searching for the Signatures of Jet-ISM Interactions in X-ray Binaries, in proceedings of A Population Explosion: The Nature \& Evolution of X-ray Binaries in Diverse Environments, 08 AIP Conf. Ser., (1010) 50.

[47] R.P. Fender, S.T. Garrington, D.J. McKay, T.W.B. Muxlow, G.G. Pooley, R.E. Spencer, A.M. Stirling, E.B. Waltman, MERLIN observations of relativistic ejections from GRS 1915+105, MNRAS 99 (304) 865 [arXiv:astro-ph/9812150].

[48] I.F. Mirabel, L.F. Rodríguez, A Superluminal Source in the Galaxy, Nature 94 (371) 46.

[49] J.C.A. Miller-Jones, M.P. Rupen, R.P. Fender, A. Rushton, G.G. Pooley, R.E. Spencer, Evidence for deceleration in the radio jets of GRS1915+105?, MNRAS 07 (375) 1087 [arXiv:astro-ph/0612211].

[50] M. Perucho, V. Bosch-Ramon, On the interaction of microquasar jets with stellar winds, A\&A 08 (482) 917 [arXiv: 0802.1134$].$

[51] A.J. Mioduszewski, M.P. Rupen, CI Camelopardalis: A Shell-shocked X-Ray Nova, ApJ 04 (615) 432 [arXiv:astro-ph/0407277].

[52] A.J. Mioduszewski, M.P. Rupen, R.M. Hjellming, G.G. Pooley, E.B. Waltman, A One-sided Highly Relativistic Jet from Cygnus X-3, ApJ 01 (553) 766 [arXiv: astro-ph/ 0102018 ].

[53] J.C.A. Miller-Jones, K.M. Blundell, M.P. Rupen, A.J. Mioduszewski, P. Duffy, A.J. Beasley, Time-sequenced Multi-Radio Frequency Observations of Cygnus X-3 in Flare, ApJ 04 (600) 368 [arXiv:astro-ph/0311277]. 
[54] V. Tudose, R.P. Fender, M.A. Garrett, J.C.A. Miller-Jones, Z. Paragi, R.E. Spencer, G.G. Pooley, M. van der Klis, A. Szomoru, First e-VLBI observations of Cygnus X-3, MNRAS 07 (375) L11 [arXiv:astro-ph/0611054].

[55] M. Ribó, J.M. Paredes, J. Moldón, J. Martí, M. Massi, The changing milliarcsecond radio morphology of the gamma-ray binary LS 5039, A\&A $\mathbf{0 8}$ (481) 17 [arXiv : 0801 . 2940].

[56] T.C. Hillwig, D.R. Gies, Spectroscopic Observations of the Mass Donor Star in SS 433, ApJ 08 (676) L37 [arXiv:0711.4348].

[57] K.M. Blundell, A.J. Mioduszewski, T.W.B. Muxlow, P. Podsiadlowski, M.P. Rupen, Images of an Equatorial Outflow in SS 433, ApJ 01 (562) L79 [arXiv: astro-ph/ 010950 4].

[58] Z. Paragi, R.C. Vermeulen, I. Fejes, R.T. Schilizzi, R.E. Spencer, A.M. Stirling, The inner radio jet region and the complex environment of SS433, A\&A 99 (348) 910 [arXiv : astro-ph/9907169].

[59] G.O. Abell, B. Margon, A kinematic model for SS433, Nature 79 (279) 701.

[60] M. Milgrom, On the interpretation of the large variations in the line positions in SS433, A\&A 79 (76) L3.

[61] A.C. Fabian, M.J. Rees, SS 433 - A double jet in action, MNRAS 79 (187) 13.

[62] P.E. Hardee, P.A. Hughes, The Effect of External Winds on Relativistic Jets, ApJ 03 (583) 116 [arXiv:astro-ph/0209593].

[63] G.E. Romero, A.T. Okazaki, M. Orellana, S.P. Owocki, Accretion vs. colliding wind models for the gamma-ray binary LS I +61 303: an assessment, A\&A 07 (474) 15 [arXiv: 0706.1320 ].

[64] D. Russell, R. Fender, E. Gallo, J.C.A. Miller-Jones, C.R. Kaiser, Jet-Powered Optical Nebulae From $X$-ray Binaries, in proceedings of VI Microquasar Workshop: Microquasars and Beyond, PoS ( MQW6 ) 059 [arXiv: astro-ph/ 0611057 ].

[65] J.A. Combi, G.E. Romero, P. Benaglia, I.F. Mirabel, The radio surroundings of the microquasar GRO J1655-40, A\&A 01 (370) L5 [arXiv:astro-ph/ 0103406 ].

[66] R.M. Hjellming, M.P. Rupen, Episodic Ejection of Relativistic Jets by the X-Ray Transient GRO:J1655-40, Nature 95 (375) 464.

[67] R. Fender, High energy astrophysics with the next generation of radio astronomy facilities, these proceedings, VII Microquasar Workshop: Microquasars and Beyond, POS ( MQW7) 055 [arXiv:0810.0951].

[68] R. Fender, B. Stappers, R. Wijers et al., LOFAR Transients and the Radio Sky Monitor, in proceedings of Bursts, Pulses and Flickering: wide-field monitoring of the dynamic radio sky, PoS (Dynamic2007) 030 [arXiv:0805.4349].

[69] F. Aharonian et al., Discovery of Very High Energy Gamma Rays Associated with an X-ray Binary, Science 05 (309) 746 [arXiv: astro-ph/ 0508298 ].

[70] F. Aharonian et al., 3.9 day orbital modulation in the TeV $\gamma$-ray flux and spectrum from the X-ray binary LS 5039, A\&A 06 (460) 743 [arXiv: astro-ph/0607192].

[71] J. Albert et al., Variable Very-High-Energy Gamma-Ray Emission from the Microquasar LS I +61 303, Science 06 (312) 1771 [arXiv: astro-ph/ 0605549 ].

[72] J. Albert et al., Very High Energy Gamma-Ray Radiation from the Stellar Mass Black Hole Binary Cygnus X-1, ApJ 07 (665) L51 [0706.1505]. 